\title{
Anomalous behavior of the excited state of the A exciton in bulk WS2
}

\author{
Jindal, Vishwas; Bhuyan, Sumi; Deilmann, Thorsten; Ghosh, Sandip
}

Published in:

Physical Review B

Link to article, DOI:

10.1103/PhysRevB.97.045211

Publication date:

2018

Document Version

Publisher's PDF, also known as Version of record

Link back to DTU Orbit

Citation (APA):

Jindal, V., Bhuyan, S., Deilmann, T., \& Ghosh, S. (2018). Anomalous behavior of the excited state of the A exciton in bulk WS2. Physical Review B, 97(4), [045211]. https://doi.org/10.1103/PhysRevB.97.045211

\section{General rights}

Copyright and moral rights for the publications made accessible in the public portal are retained by the authors and/or other copyright owners and it is a condition of accessing publications that users recognise and abide by the legal requirements associated with these rights.

- Users may download and print one copy of any publication from the public portal for the purpose of private study or research.

- You may not further distribute the material or use it for any profit-making activity or commercial gain

- You may freely distribute the URL identifying the publication in the public portal

If you believe that this document breaches copyright please contact us providing details, and we will remove access to the work immediately and investigate your claim 


\title{
Anomalous behavior of the excited state of the $A$ exciton in bulk $\mathrm{WS}_{2}$
}

\author{
Vishwas Jindal, ${ }^{1}$ Sumi Bhuyan, ${ }^{1}$ Thorsten Deilmann, ${ }^{2, *}$ and Sandip Ghosh ${ }^{1, \dagger}$ \\ ${ }^{1}$ Department of Condensed Matter Physics and Materials Science, Tata Institute of Fundamental Research, Mumbai 400005, India \\ ${ }^{2}$ Center for Atomic-Scale Materials Design, Department of Physics, Technical University of Denmark, DK-2800 Kongens Lyngby, Denmark
}

(Received 17 December 2017; revised manuscript received 18 January 2018; published 31 January 2018)

\begin{abstract}
Results of optical spectroscopy studies on bulk $2 \mathrm{H}$-WS 2 at energies close to its direct band gap are presented. Reflectance and absorption measurements at low temperature show only one dominant feature due to the $A$ exciton of bulk $\mathrm{WS}_{2}$ at $\sim 2.02 \mathrm{eV}$. However, a laser-modulated photoreflectance spectrum looks quite different, revealing a second even stronger feature $A^{*} \sim 62 \mathrm{meV}$ above $A$. The relative intensity of these two features is shown to change significantly in a lateral electroreflectance measurement with electric field applied perpendicular to the $\mathbf{c}$ axis of $\mathrm{WS}_{2}$. The experimental results are analyzed by comparison with many-body perturbation theory calculations, including the solutions of the Bethe-Salpeter equation. $A^{*}$ is identified as the first excited state of the $A$ exciton, that is, $A(n=2)$. The anomalous behavior of $A^{*}$ is explained by its distinct wave function spread along the $\mathbf{c}$ axis, the direction of weak van der Waals bonding, which makes it more susceptible to perturbations. Our $a b$ initio calculations suggest that the $A$ exciton in the ground state has a two-dimensional (2D) nature with a large binding energy $E_{\mathrm{b}}$, in fair agreement with $E_{\mathrm{b}} \sim 90 \pm 20 \mathrm{meV}$ estimated from a temperature-dependent reflectance study. The applicability of the $2 \mathrm{D}$ hydrogenic Wannier-Mott model for the exciton spectrum of a layered semiconductor like bulk $\mathrm{WS}_{2}$ is discussed.
\end{abstract}

DOI: 10.1103/PhysRevB.97.045211

\section{INTRODUCTION}

Recent studies on transition-metal dichalcogenide (TMDC) semiconductors [1] such as $\mathrm{WS}_{2}$ are motivated by the discovery of novel properties in them that also hold promise for future electronic device applications. These layered materials, held together by weak van der Waals forces perpendicular to the layers, can exist in stable monolayer form. One finds that $\mathrm{WS}_{2}$ in the monolayer two-dimensional (2D) form is a direct band gap semiconductor [2], although it has an indirect band gap in bulk crystal form. The monolayer shows a strong coupling of spin and valley degrees of freedom [3] and has very large exciton and trion binding energies [4,5]. The large exciton binding energy $E_{\mathrm{b}}$ in monolayer $\mathrm{WS}_{2}$ also makes it interesting for room-temperature exciton-polariton condensation studies and related applications [6]. The properties of excitons in these materials [7] in general seem quite different from conventional direct band gap group-III-V semiconductors. It has been shown that due to the rapid change in the dielectric environment across a monolayer, the energy spectrum of excitons can be dramatically different from what one expects from a $2 \mathrm{D}$ hydrogenic Wannier-Mott exciton model [8]. Earlier, it was thought that even in bulk TMDC crystals, excitons may have $2 \mathrm{D}$ character [9] originating from the large anisotropy in their properties between directions parallel and perpendicular to the layers, the latter defining the $\mathbf{c}$ axis. Recent studies also support this. For instance, exciton polarizability measurements show layer-independent behavior, indicating strong localization of electron and hole wave functions in individual layers of a

\footnotetext{
*thorsten.deilmann@wwu.de

†sangho10@tifr.res.in
}

multilayer sample [10]. Two-dimensional behavior of excitons in bulk has also been inferred from analyzing the optical absorption spectrum [11] and theoretical studies [12]. As such the electronic band structure around the fundamental band gap in a semiconductor determines the device characteristics. In monolayer $\mathrm{WS}_{2}$ this gap, around which the excitons arise, is at the $K$ point of the Brillouin zone [13], which is also where the direct band gap arises in bulk $\mathrm{WS}_{2}$ [14]. For all these reasons it is important to understand the electronic structure and exciton spectrum around the direct band gap of bulk $\mathrm{WS}_{2}$.

Absorption studies on bulk $\mathrm{WS}_{2}$ around its direct band gap show two dominant peaks, labeled $A$ and $B$, which arise from ground-state exciton transitions involving two spin-orbit split valence and conduction bands at the $K$ point of the Brillouin zone [14]. The lower-energy $A$ feature defines the direct excitonic band gap. These materials have two main allotropic crystal forms, $2 H$ and $3 R$, the former being more common. It was thought that the exciton spectrum in the $3 R$ form follows the $2 \mathrm{D}$ hydrogenic model and was three-dimensional-like in $2 H$ [9]. A very weak feature seen between $A$ and $B$ in $3 R-\mathrm{WS}_{2}$ was early on attributed [15] to the first excited-state transition of $A$, that is, $A(n=2)$ in a $2 \mathrm{D}$ hydrogenic model. Other studies on similar materials such as bulk $2 \mathrm{H}-\mathrm{MoS}_{2}$ also showed additional features at energies between the $A$ and $B$ features which were also attributed to higher excited-state exciton transitions, but these identifications could not be reconciled fully with conventional hydrogenic exciton models [16]. Recent studies [17] have suggested that these weak features in $2 \mathrm{H}-\mathrm{MoS}_{2}$ could be altogether different exciton transitions at the $H$ point of the Brillouin zone of bulk $2 \mathrm{H}-\mathrm{MoS}_{2}$, but the matter is not settled. Such weak features in $2 \mathrm{H}$-WS 2 at energies above the $A$ exciton feature have also been reported in recent reflectance contrast and modulated reflectance spectroscopy studies, but 
there are competing claims of them being either $A(n=2)$ or $H$-point transitions [18-20]. Here we present results of a study on bulk $2 \mathrm{H}-\mathrm{WS}_{2}$ around its direct band gap combining several complementary optical spectroscopy techniques. We show that an extra spectral feature, even stronger than the $A$-exciton feature, can arise in a photomodulated reflectance spectrum of bulk $2 \mathrm{H}-\mathrm{WS}_{2}$. We try to verify its origin through additional direct electromodulated reflectance measurements, spectral line shape analysis, and comparison with many-body perturbation-theory-based band structure calculations as well as two-particle excitations based on solving the Bethe-Salpeter equation. Temperature-dependent reflectance studies are used to verify the theoretically estimated $E_{\mathrm{b}}$ values. Finally, we discuss the implication of these results for the hydrogenic model for excitons in bulk $2 \mathrm{H}-\mathrm{WS}_{2}$.

\section{EXPERIMENTAL DETAILS}

The $C$-plane-oriented $2 \mathrm{H}$ - $\mathrm{WS}_{2}$ flakes, obtained from HQGraphene, were cleaned by exfoliation and transferred onto sapphire substrates. Their $2 H$ crystal structure was verified using $\mathrm{x}$-ray diffraction [21]. Sapphire was chosen as a substrate because its high thermal conductivity enabled efficient cooling of the sample; also, being transparent and insulating, it aided transmission and lateral electroreflectance (LER) measurements. The samples were cooled using a continuous-flow liquid-He cryostat with optical access. Measurements above room temperature were performed in a vacuum chamber with optical access and arrangement for sample heating, temperature sensing, and control. The sample thicknesses were typically $\sim 0.5 \mu \mathrm{m}$, except for transmission measurements, which required thinner samples of $\sim 0.05 \mu \mathrm{m}$. The exfoliated flakes had a small area, and therefore all optical measurements were done under a microscope arrangement using a $10 x$ objective with a probe beam spot size of $30 \times 140 \mu \mathrm{m}^{2}$. The microscope arrangement is coupled to a $75-\mathrm{W}$ xenon lamp and a grating monochromator combination which provided the probe beam with a band pass of $\sim 1.5 \mathrm{meV}$. For the photoreflectance (PR) measurements a 532-nm Nd-YAG laser was used as the pump beam. For the LER measurements aluminum electrodes with a spacing of $100 \mu \mathrm{m}$ were thermally evaporated onto the sample, and a sinusoidal voltage with $50 \mathrm{~V}$ amplitude was applied across them. A GaAs photomultiplier tube was used for detection, and in all cases phase-sensitive signal measurement was performed using a lock-in amplifier.

\section{EXPERIMENTAL RESULTS}

Figures 1(a) and 1(b) show the reflectance $R$ and absorption $\alpha$ spectrum of bulk $2 \mathrm{H}-\mathrm{WS}_{2}$ at $5 \mathrm{~K}$. We have simulated the $R$ spectrum by considering the exciton contribution to the dielectric function $\varepsilon(E)$ as a function of energy $E$ as given by the Lorentz oscillator model [22]

$$
\varepsilon(E)=\left(n_{b}+\iota k_{b}\right)^{2}+\sum_{j} \frac{C_{j}}{E_{0 j}^{2}-E^{2}-\iota \Gamma_{j} E} .
$$

Here $n_{b}$ and $k_{b}$ represent the real and imaginary parts of the background refractive index. $E_{0 j}$ and $\Gamma_{j}$ are the transition energy and broadening parameter, while $C_{j}$ is a parameter proportional to the oscillator strength of the $j$ th exciton

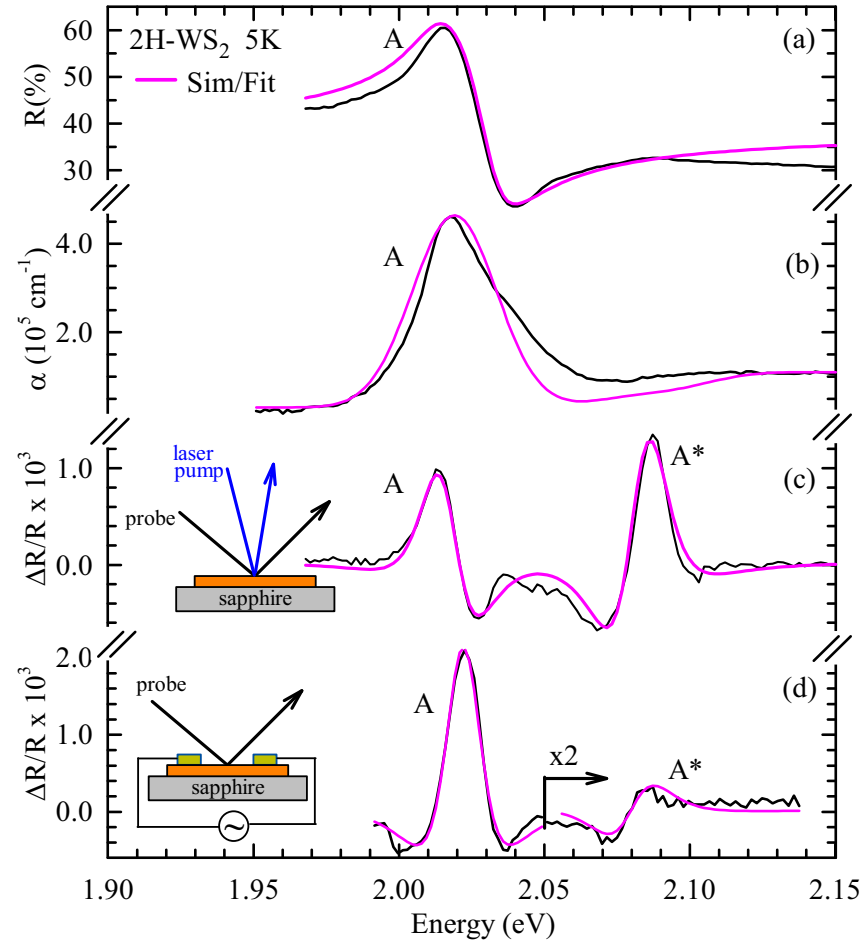

FIG. 1. (a) Reflectance $R$, (b) absorption $\alpha$, (c) photoreflectance (PR), and (d) lateral electroreflectance (LER) spectrum of bulk $\mathrm{WS}_{2}$ flakes at low temperature. The fitted/simulated line shapes are also shown. The insets in (c) and (d) are schematics of the PR and LER measurement geometry; the actual measurements used the microscope arrangement described in the text.

transition. We find that by considering a single transition we are able to fairly reproduce the measured spectrum with $E_{0}=2.012 \mathrm{eV}$. Also the absorption spectrum shows one dominant feature at $2.018 \mathrm{eV}$. These features arise from the ground-state $A$ exciton transition in bulk $\mathrm{WS}_{2}$ [14]. The $B$ exciton transition is at a much higher energy of $2.433 \mathrm{eV}$ [21]. We also studied the evolution of the $A$ exciton resonance in $R$ as a function of temperature, which we shall show and discuss later. Figure 1(c) shows the PR spectrum of the sample where in addition to the $A$ feature one finds a stronger feature labeled $A^{*}$. To estimate the transition energies in PR we have fitted to these feature Aspnes's derivative functional form line shape function. We used a modified set of fitting parameters [21] which makes it easier to fit and compare feature strengths [23]. The transition energies for $A$ and $A^{*}$ in PR were found to be 2.017 and $2.082 \mathrm{eV}$, respectively, with the strength of $A^{*}$ being $\sim 1.7$ times larger than that of $A$. Figure 1(d) shows the LER spectrum of a sample where, again, one finds the extra $A^{*}$ feature, although now much reduced in strength. Again fitting Aspnes's line shape function, the transition energies of $A$ and $A^{*}$ in LER were found to be 2.022 and $2.080 \mathrm{eV}$, respectively, with the strength of $A^{*}$ about $1 / 6.7(15 \%)$ that of $A$. The measurement-technique-dependent differences in estimated $E_{0}$ values, which have been seen before in other [17] systems, are small and can be neglected here. Thus in bulk $\mathrm{WS}_{2}$ there is another transition $A^{*}$ which is $62 \pm 3 \mathrm{meV}$ above the $A$ exciton transition. If $A^{*}$ corresponds to the $A(n=2)$ transition of a conventional 2D hydrogenic model [4], then 


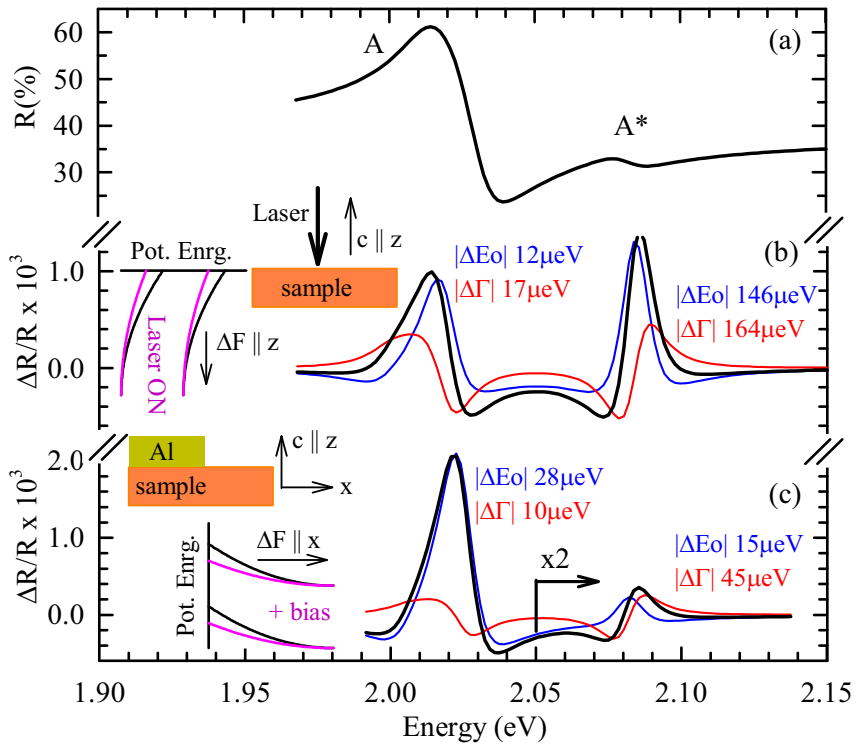

FIG. 2. Simulated (a) $R$, (b) PR, and (c) LER spectrum of bulk $\mathrm{WS}_{2}$ at $5 \mathrm{~K}$. In (b) and (c) the $\Delta E_{0}$ and $\Delta \Gamma$ values used in the simulation are shown. The thin lines represent their contribution to the spectrum when considered individually. The insets schematically show the potential profiles and dominant electric field change $\Delta F$ directions in PR and LER relative to the $\mathbf{c}$ axis of $\mathrm{WS}_{2}$.

its oscillator strength, which goes as $1 /(n-0.5)^{3}$, should be $1 / 27(3.7 \%)$ that of $A$. The fact that it is larger than $A$ in PR and then becomes relatively much weaker in LER is clearly an anomalous behavior. To understand this we further explore the PR and LER results through simulations.

In modulation spectroscopy measurements like PR and LER, typically, there is a change in the net electric field which affects the exciton. In the case of PR the electric field change is due to the separation of laser-generated free electrons and holes, which reduces the built-in fields at the surface/interfaces of a semiconductor and in the case of LER directly due to the applied field [24]. The consequent change in an $R$ spectrum [25] can be quantified in terms of changes in the parameters $E_{o}, \Gamma$, and $C$ defining the exciton contribution to $\varepsilon(E)$ in Eq. (1). In Fig. 2(a) we show a simulated $R$ spectrum, where in addition to the $A$ transition we have considered another transition to represent $A^{*}$ with a strength of 5\% that of $A$ based on theoretical results that will be discussed later. This gives rise to a weak kink on the high-energy side of the $A$ feature in the simulated $R$ spectrum. Next we change the parameters to $E_{0}+\Delta E_{0}$ and $\Gamma+\Delta \Gamma$ and calculate $R^{\prime}$ and thereafter the modulated reflectance spectrum as $\Delta R / R=\left[R^{\prime}-R\right] / R$. We did not consider $\Delta C$ change since it gives rise to long tails not observed in the experiments and also because, as will be shown later, $E_{\mathrm{b}}$ is large, which ensures a stable oscillator strength. By varying the parameters $\Delta E_{0}$ and $\Delta \Gamma$ we have simulated the measured PR and LER spectrum, which are shown in Figs. 2(b) and 2(c). The modulation strengths $\Delta E_{0}$ and $\Delta \Gamma$ are mentioned alongside each transition. One finds that in PR, $\Delta E_{0}$ and $\Delta \Gamma$ values are larger by a factor of $\sim 10$ for $A^{*}$ compared to $A$. In LER, too, the total modulation strength is larger for $A^{*}$ but by a smaller factor. In PR and LER measurements, the signal strength is not just proportional to the oscillator

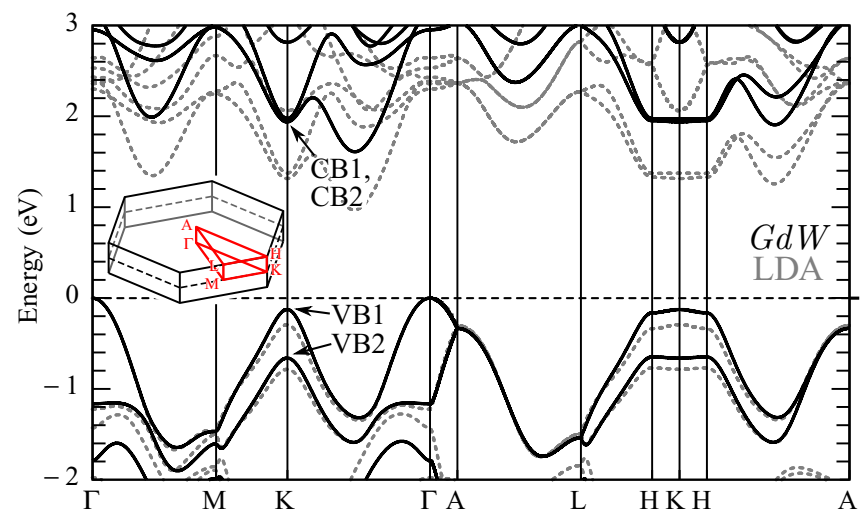

FIG. 3. Electronic band structure of bulk $\mathrm{WS}_{2}$ using DFT (LDA) (gray dashed lines) and the many-body calculation in the $G d W$ approximation (black lines). Both band structures are aligned at the VB maximum. The inset shows the first Brillouin zone of bulk $\mathrm{WS}_{2}$ with the irreducible band path highlighted in red.

strength but also depends on the modulation strength [25]. We therefore infer that while the oscillator strength of $A^{*}$ is much weaker than that of $A$, it is subjected to stronger modulation than $A$ and also that the relative modulation experienced by $A^{*}$ is much stronger in PR than in an LER measurement. In order to understand these results we performed theoretical band structure calculations which are presented next.

\section{THEORY RESULTS}

We theoretically investigated the electronic and optical properties of the bulk $\mathrm{WS}_{2}$ from first principles by employing the experimental lattice parameters [26]. We evaluate the band structure within density functional theory (DFT) using the local-density approximation (LDA) including spin-orbit interaction and many-body perturbation theory $(G W$ within the LDA $+G d W$ approximation [27]) and use the Bethe-Salpeter equation (BSE) to calculate the optical absorption spectra as well as the properties of the excitons [28].

The resulting band structures are shown in Fig. 3. The DFT calculation is well converged with a mesh of $12 \times 12 \times 3$ points; for $G d W$ a slightly larger mesh of $21 \times 21 \times 4$ points is employed. We find an indirect gap of $1.61 \mathrm{eV}$ between the $\Gamma$ and $\Sigma$ (minimum of the $K-\Gamma$ line) points of the Brillouin zone, shown as an inset in Fig. 3. The minimal direct gap of $2.07 \mathrm{eV}$ is observed exactly at the $K$ point. In comparison to DFT (LDA) where the gaps were $0.97 \mathrm{eV}$ (indirect) and $1.61 \mathrm{eV}$ (direct), the gaps are distinctly larger in $G d W$, which reveals its many-body nature and shows reasonable agreement with previous results [29]. Focusing on the $H$ point, we find that it is a local potential minimum for carriers in the $A-H-L$ plane but not along the $H-K$ direction (i.e., $\| \mathbf{c}$ axis in real space). The direct gap at $H$ is $2.11 \mathrm{eV}$ in $G d W(1.67 \mathrm{eV}$ in LDA), and thus the difference relative to the gap at $K$ is $40 \mathrm{meV}$ (60 meV in LDA). We note that all states/bands are doubly degenerate and the bands can be roughly attributed to only one layer [e.g., for the valence band (VB) the contribution from farther layers is less than 6\%]. At the $K$ point carriers in the lowest-energy conduction band CB1 and next-higher-energy CB2 have opposite spins, which is also true for valence bands 

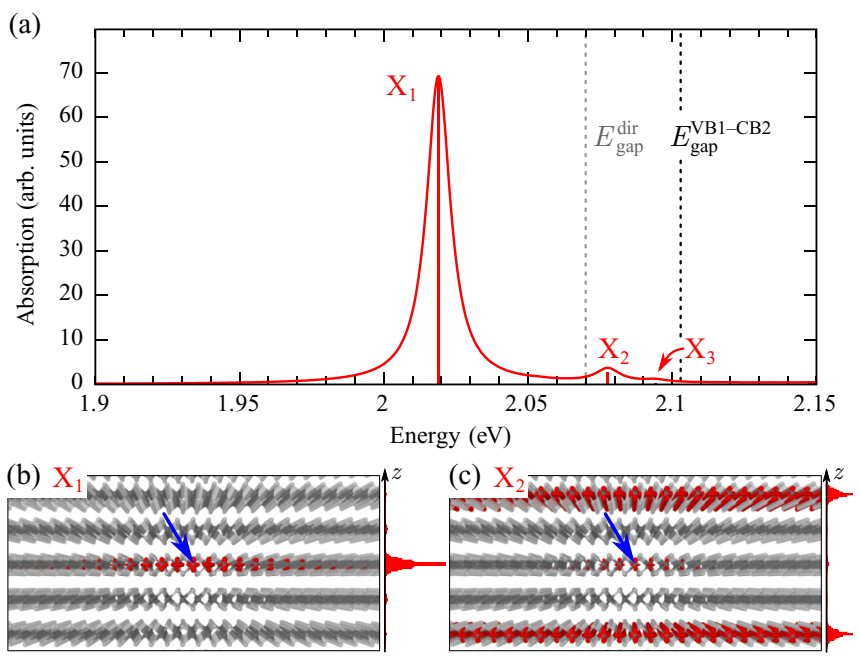

FIG. 4. (a) $G d W$-BSE absorption spectra in bulk $\mathrm{WS}_{2}$ calculated using a $21 \times 21 \times 6$ mesh. The direct gap $E_{\text {gap }}^{\text {dir }}$ between VB1 and CB 1 and that between VB1 and CB2 are marked. Electron probability $|\Psi|^{2}$ and its projection along $z$ is shown in (b) for $X_{1}$ and (c) for $X_{2}$. The position of the fixed hole is marked by blue arrows.

VB1 and VB2. Also the spin alignment is opposite in adjacent layers.

Next, we investigate the optical properties by including electron-hole interactions and solving the BSE [28]. We include four VBs and eight CBs, that is, two VBs and four CBs per layer. While the $G W / G d W$ calculations already require a fine mesh, the convergence of BSE results is typically even more demanding. All meshes in our calculation include the $K$ and $H$ points, and we have verified the convergence of exciton energy and oscillator strengths [21]. We find three optically bright excitons at energies close to the direct band gap [Fig. 4(a)]. We label them $X_{1}, X_{2}$, and $X_{3}$, wherein the lowest-energy $X_{1}$ exciton at $2.018 \mathrm{eV}$ is by far the strongest $\left(X_{2} / X_{1}\right.$ strength $\sim 5 \%$ and $X_{3} / X_{1}$ strength $\left.\sim 0.5 \%\right) . X_{2}$ and $X_{3}$ lie about 60 and $80 \mathrm{meV}$ above $X_{1}$, respectively. We ignore $X_{3}$ hereafter given its weak strength, which makes it practically undetectable in experiment.

All exciton states have a degeneracy of 2 due to the degenerate bands and another 2 due to symmetry of $\pm K$. Further insights into the properties of the excitons can be gained from their band contributions and their localization and extent in $k$ space as well as in real space. As for band contributions, $X_{1}$ and $X_{2}$ show similar characteristics. We find that the transitions stem mostly from the highest valence band VB1 to the second-lowest conduction band CB2 [see labels in Fig. 3(a)]. The possible lowest-energy direct transition, involving CB1 and VB1, is optically dark, similar to the case of monolayer $\mathrm{WS}_{2}, \mathrm{MoS}_{2}$, and $\mathrm{WSe}_{2}$ [30-33]. The CB1-CB2 splitting is about $30 \mathrm{meV}$ at the $K$ point, making the VB1-CB2 direct "bright" band gap $\sim 2.10 \mathrm{eV}$. Since the $X_{1}$ exciton at $2.018 \mathrm{eV}$ stems mostly from VB1 and CB2, its theoretical binding energy, relative to the gap of $2.10 \mathrm{eV}$, becomes $E_{\mathrm{b}}$ $\sim 85 \mathrm{meV}$.

Significant contributions to both excitons $X_{1}$ and $X_{2}$ are only found along the $K-H$ line [21] centered around the $k_{z}=0$ plane, that is, at the $K$ point. For $X_{1}$ the $k_{z}$ contributions decrease away from $k_{z}=0$, while for $X_{2}$ the contribution becomes more complex and shows a node on this line (two nodes for $X_{3}$, etc.). This means that we can identify $X_{2}$ as the first excited state ( $n=2$ of a Rydberg-like series), which is not particularly located around the $H$ point. Indeed, we find no low-energy bright exciton that is prominently located at the $H$ point.

In addition to the properties in reciprocal space it is also instructive to look at the exciton's spread in real space. To visualize the six-dimensional (three dimensions of the electron, three of the hole) exciton wave function we fix the hole in one layer and calculate the electron probability $|\Psi|^{2}$. To illustrate its distribution we integrate over the remaining directions. Figures 4(b) and 4(c) show such plots for the $X_{1}$ and $X_{2}$ excitons in the real-space $x-z$ plane. We find that for $X_{1}$ the electron is mostly confined to the same layer as the hole. $X_{2}$ is quite different in that, close to the hole, only a small contribution is found in the same layer, while a maximum is observed in the next but one layer along $z$. Thus $X_{2}$ is much more spread out along the $z$ axis (i.e., $\|$ c) compared to $X_{1}$.

\section{DISCUSSION}

The similarity of the energy and strength of the bright $X_{1}$ exciton around the $K$ point as found above clearly identifies it with the $A$ exciton seen in our measurements. Although the $H$-point and $K$-point band gap difference is similar to the $A-A^{*}$ energy separation, there are no indications of any low-energy excitons predominantly located at the $H$ point in the calculations. This is essentially due to the absence of a potential minimum for carriers at the $H$ point along all $k$ directions. Given that the energy separation of $X_{1}$ and $X_{2}$ matches that of $A$ and $A^{*}$ and also that $X_{2}$ is much weaker than $X_{1}$, much like the barely seen $A^{*}$ in the $R$ spectrum, we identify $A^{*}$ with $X_{2}$. We note that the agreement between experiment and theory is surprisingly good. Since $X_{1}$ and $X_{2}$ originate from the same bands, $A^{*}$ can be considered an excited-state $A(n=2)$ transition. The simulated absorption spectrum in Fig. 1(b) considered two exciton transitions, with their relative oscillator strengths and energy spacing determined by $X_{1}$ and $X_{2}$ above, plus a step function to account for band-toband transition beyond the band gap, and is convolved with a Gaussian to account for broadening. It agrees fairly well with the measurement. The above identification of $A^{*}$ as the effective $A(n=2)$ gives a clue to the relative strengths of the $A$ and $A^{*}$ features in PR and LER measurements as follows. The crystal is held together in the direction $\| \mathbf{c}$ axis of $\mathrm{WS}_{2}$, mostly by weak van der Waals forces, and the wave function for the $A(n=2)$ exciton state has a considerable spread along this direction, unlike that for $A$, as seen in Figs. 4(b) and 4(c). Thus a perturbation in general is likely to modulate $A(n=2)$ much more than $A$. If the perturbation is an electric field $\| \mathbf{c}$, then $A(n=2)$ will be modulated even more strongly. In PR the laser is incident $\| \mathbf{c}$, and its absorption will change the built-in field along this direction, as shown in the inset of Fig. 2(b). Thus in PR we should get very strong modulation of $A(n=2)$. However, if the field change is $\perp \mathbf{c}$, then it may not affect $A(n=2)$ as much. In fact, it is to test this idea that we performed the LER measurements where the field change, as indicated in the inset of Fig. 2(c), is mostly $\perp$ c. We saw 

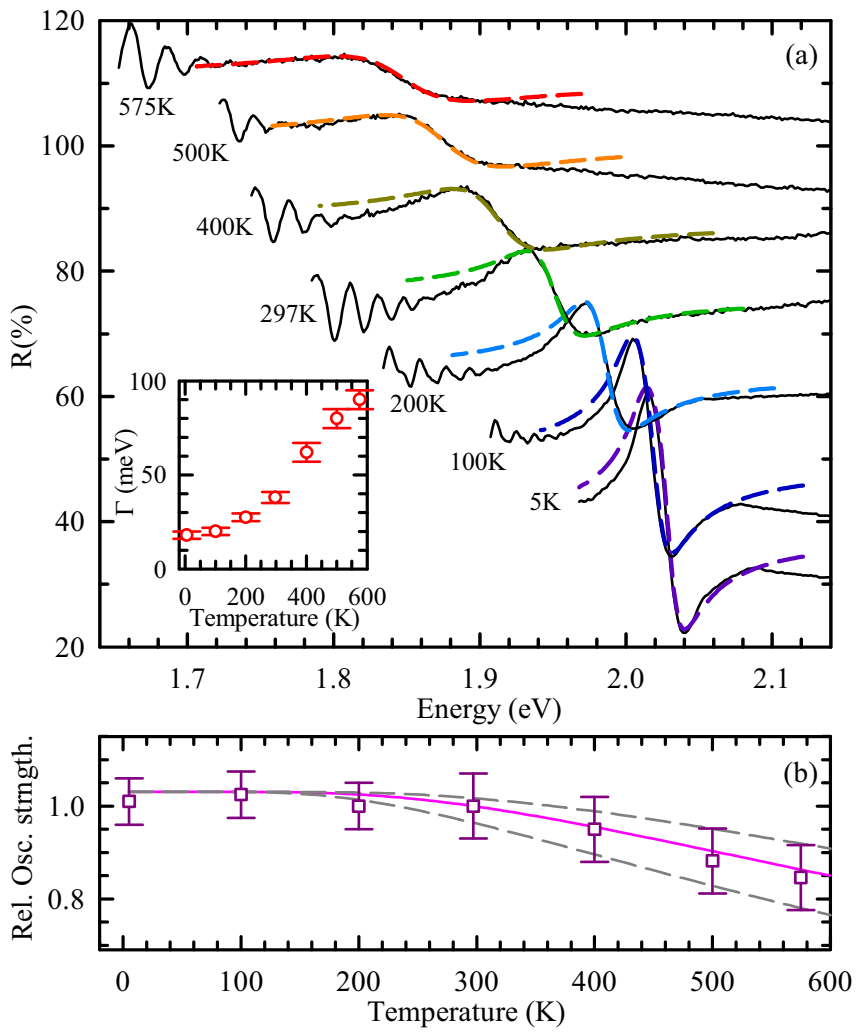

FIG. 5. (a) Temperature dependence of the reflectance spectrum of bulk $\mathrm{WS}_{2}$ showing the variation in the $A$ exciton feature's strength. The plots are vertically shifted for clarity. The dashed lines are simulations based on Eq. (1). The inset shows the change in the broadening parameter $\Gamma$ in Eq. (1). (b) Temperature dependence of the parameter $C$ in Eq. (1), which is proportional to oscillator strength. The values are relative to $C$ at $297 \mathrm{~K}$. The solid line represents expected decay in the relative oscillator strength with temperature for $E_{\mathrm{b}}=90 \mathrm{meV}$ and the upper and lower dashed lines are for $E_{\mathrm{b}}=90 \pm 20 \mathrm{meV}$.

that in LER the total modulation strength for $A^{*}$, although still larger than that for $A$, is considerably reduced relative to its value in PR, clearly supporting this idea. We note in passing that we have not observed any indication of a bright interlayer exciton (as, for example, in $\mathrm{MoTe}_{2}$ [19] and $\mathrm{MoSe}_{2}$ [34]) in bulk $\mathrm{WS}_{2}$ in our theoretical calculations. An interlayer exciton would also have been subjected to strong modulation for field changes parallel to the $\mathbf{c}$ axis; however, we see no additional feature in PR other than the one identified as $A(n=2)$ here.

We next investigate the theoretically predicted large $E_{\mathrm{b}}$ value. As mentioned earlier, the VB1-CB1 transition is optically dark, and the $A$ exciton transition involves carriers in VB1-CB2 with $E_{\mathrm{b}} \sim 85 \mathrm{meV}$. We tried to experimentally estimate $E_{\mathrm{b}}$ using the decay of the exciton oscillator strength with an increase in temperature by studying the temperature dependence of the $R$ spectrum, as shown in Fig. 5(a). The oscillatory features seen in Fig. 5(a) at subband gap energies are due to optical interference effects and have been ignored in the analysis. Consider that once an exciton is thermally excited to the continuum edge, the electron and hole are free to move independently in the lattice, get trapped by defects, and recombine nonradiatively, thereby leading to a loss of excitons. This would result in a reduced value of $C$ in Eq. (1) which is proportional to the oscillator strength, in effect the number of excitons. In the simplest analysis considering this to be a two-level system, neglecting other excited states since their oscillator strengths are negligible compared to the ground state, one can write the probability $p$ of excitation to the continuum state from the ground state at temperature $T$ as $p=\exp \left[-E_{\mathrm{b}} / k_{B} T\right]$, where $k_{B}$ is the Boltzmann constant. Then the number of excitons that remain in the ground state is proportional to $1-p=1-\exp \left[-E_{\mathrm{b}} / k_{B} T\right]$, and the value of $C$ should be proportional to this. We have simulated the exciton-dominated part of the $R$ spectrum at different temperatures by varying the parameters in Eq. (1) to match the measured spectrum. The values of $C$ thus obtained are plotted in Fig. 5(b). One finds that although there seems to be a significant decrease in the $A$ exciton feature's strength with an increase in temperature, the value of $C$ actually does not decay much. The changes occur mainly due to a large increase in the broadening parameter $\Gamma$ from $18 \mathrm{meV}$ at $5 \mathrm{~K}$ to about $90 \mathrm{meV}$ at $575 \mathrm{~K}$, as shown in the inset of Fig. 5(a). The weak decay of $C$ indicates a fairly large $E_{\mathrm{b}}$. However, there are large uncertainties in the estimated $C$ values arising from the accuracy of spectral response (magnitude) corrections and the fact that $C$ and $\Gamma$ values are correlated; an increase in one can nullify the effect of the increase in the other. The simulated lines in Fig. 5(b), based on the above thermal decay model, indicate that $E_{\mathrm{b}} \sim 90 \pm 20 \mathrm{meV}$, which is close to the theoretical estimate of $85 \mathrm{meV}$, although one must note that the experimental error bars are large. Thus the "bright" band gap of bulk $2 \mathrm{H}-\mathrm{WS}_{2}$ obtained through experiment comes to $2.11 \pm 0.02 \mathrm{eV}$. Note that in the above analysis we did not consider any influence of CB1 since, if it existed, the $A$ exciton oscillator strength should have increased with the increase in temperature as seen for the nonresonantly excited photoluminescence signal strength in monolayer $\mathrm{WSe}_{2}$ [30,35]. CB1 can be excluded because the optically resonantly excited carriers in CB2 in a reflectance measurement cannot relax efficiently to $\mathrm{CB} 1$ due to its opposite spin orientation.

Finally, we discuss the appropriateness of the hydrogenic model in describing excitons in bulk $2 \mathrm{H}-\mathrm{WS}_{2}$. We note that the oscillator strength ratio of the transitions identified as $A(n=2)$ and $A$ here was theoretically found to be $\sim 5 \%$ from the BSE analysis. This is close to what one would expect $(3.7 \%$, as mentioned earlier) from a $2 \mathrm{D}$ hydrogenic model. If we consider the energy spectrum, then in the $2 \mathrm{D}$ hydrogenic model it should go as $\mathrm{Ry}_{2 \mathrm{D}}^{*} /(n-0.5)^{2}$ where $\mathrm{Ry}^{*}$ is the effective Rydberg constant. From our experimental data one gets $\mathrm{Ry}_{2 \mathrm{D}}^{*}=(9 / 32)\left[E_{0, n=2}-E_{0, n=1}\right]$ and therefore $E_{\mathrm{b}}=$ $4 \mathrm{Ry}_{2 \mathrm{D}}^{*} \sim 70 \mathrm{meV}$. This is lower than our measured average $E_{\mathrm{b}}$ value but is still within limits set by measurement errors. In the three-dimensional (3D) model $\mathrm{Ry}_{3 \mathrm{D}}^{*}=(4 / 3)\left[E_{0, n=2}-\right.$ $\left.E_{0, n=1}\right]$, so $E_{\mathrm{b}}=\mathrm{Ry}_{3 \mathrm{D}}^{*} \sim 85 \mathrm{meV}$, which is in slightly better agreement with experiment. However, the oscillator strength ratio in $3 \mathrm{D}$ goes as $1 / n^{3}$, making $A(n=2) 12.5 \%$ of $A$ in strength, much larger than what is found experimentally and from GW-BSE analysis. The true picture in terms of the spread of the wave function along the $\mathbf{c}$ axis, which was crucial for our understanding of the relative changes in the modulation strengths between $A$ and $A(n=2)$ in PR and LER, cannot be captured by the $2 \mathrm{D}$ hydrogen-atom model. 


\section{CONCLUSION}

In conclusion we have shown that in a modulation spectroscopy measurement on bulk $\mathrm{WS}_{2}$ one can observe a feature on the high-energy side of the $A$ exciton transition whose magnitude relative to $A$ can change significantly depending on the measurement technique. By comparison with theoretical $G W$-BSE calculations we identified this feature as the signature of an effective $A(n=2)$ excited-state transition and not an $H$-point exciton transition. Furthermore, the theoretical study indicates that the lowest-energy direct transition at the $K$ point is optically dark. The bright $A$ exciton is associated with the next-higher-energy direct transition involving VB1-CB2 and has a large binding energy of about $90 \mathrm{meV}$, which is more than three times $k_{B} T \sim 26 \mathrm{meV}$ at room temperature. Excitons in bulk $\mathrm{WS}_{2}$ show many characteristics of a $2 \mathrm{D}$ system, some of which can be roughly described by a 2D hydrogenic model. However, other properties, such as the wave function spread along the $\mathbf{c}$ axis and its consequences, cannot be understood on the basis of a simple 2D hydrogenic model.

\section{ACKNOWLEDGMENTS}

The authors thank V. Sugunakar for help with instrumentation and A. Arora and L. Wirtz for very useful discussions. T.D. acknowledges financial support from the Villum Foundation.
[1] A. V. Kolobov and J. Tominaga, Two-Dimensional TransitionMetal Dichalcogenides (Springer, Berlin, 2016).

[2] W. Zhao, Z. Ghorannevis, L. Chu, M. Toh, C. Kloc, P. H. Tan, and G. Eda, ACS Nano 7, 791 (2013).

[3] T. Scrace, Y. Tsai, B. Barman, L. Schweidenback, A. Petrou, G. Kioseoglou, I. Ozfidan, M. Korkusinski, and P. Hawrylak, Nat. Nanotechnol. 10, 603 (2015).

[4] B. Zhu, X. Chen, and X. Cui, Sci. Rep. 5, 9218 (2015).

[5] G. Plechinger, P. Nagler, A. Arora, R. Schmidt, A. Chernikov, A. G. del Águila, P. C. M. Christianen, R. Bratschitsch, C. Schuller, and T. Korn, Nat. Commun. 7, 12715 (2016).

[6] L. C. Flatten, Z. He, D. M. Coles, A. A. P. Trichet, A. W. Powell, R. A. Taylor, J. H. Warner, and J. M. Smith, Sci. Rep. 6, 33134 (2016).

[7] G. Wang, A. Chernikov, M. M. Glazov, T. F. Heinz, X. Marie, T. Amand, and B. Urbaszek, [Rev. Mod. Phys. (to be published)], arXiv:1707.05863.

[8] A. Chernikov, T. C. Berkelbach, H. M. Hill, A. Rigosi, Y. Li, O. B. Aslan, D. R. Reichman, M. S. Hybertsen, and T. F. Heinz, Phys. Rev. Lett. 113, 076802 (2014).

[9] A. R. Beal, J. C. Knights, and W. Y. Liang, J. Phys. C 5, 3540 (1972).

[10] J. Klein, J. Wierzbowski, A. Regler, J. Becker, F. Heimbach, K. Muller, M. Kaniber, and J. J. Finley, Nano Lett. 16, 1554 (2016).

[11] N. Saigal, V. Sugunakar, and S. Ghosh, Appl. Phys. Lett. 108, 132105 (2016).

[12] A. Molina-Sanchez, D. Sangalli, K. Hummer, A. Marini, and L. Wirtz, Phys. Rev. B 88, 045412 (2013).

[13] G. B. Liu, D. Xiao, Y. Yao, X. Xude, and W. Yao, Chem. Soc. Rev. 44, 2643 (2015).

[14] R. Coehoorn, C. Haas, and R. A. de Groot, Phys. Rev. B 35, 6203 (1987)

[15] A. R. Beal and W. Y. Liang, J. Phys. C 9, 2459 (1976).

[16] E. Fortin and F. Raga, Phys. Rev. B 11, 905 (1975).

[17] N. Saigal and S. Ghosh, Appl. Phys. Lett. 106, 182103 (2015).

[18] M. R. Molas, K. Nogajewski, A. O. Slobodeniuk, J. Binder, M. Bartosa, and M. Potemski, Nanoscale 9, 13128 (2017).
[19] A. Arora, M. Drüppel, R. Schmidt, T. Deilmann, R. Schneider, M. R. Molas, P. Marauhn, S. M. de Vasconcellos, M. Potemski, M. Rohlfing, and R. Bratschitsch, Nat. Commun. 8, 639 (2017).

[20] J. Kopaczek, M. P. Polak, P. Scharoch, K. Wu, B. Chen, S. Tongay, and R. Kudrawiec, J. Appl. Phys. 119, 235705 (2016).

[21] See Supplemental Material at http://link.aps.org/supplemental/ 10.1103/PhysRevB.97.045211 for information on x-ray diffraction results, absorption spectrum at higher energies, the temperature dependence of transition energies, spectral line shape fitting, and details of theoretical calculations.

[22] F. Wooten, Optical Properties of Solids (Academic, New York, 1972).

[23] S. Ghosh and H. T. Grahn, J. Appl. Phys. 90, 500 (2001).

[24] O. J. Glembocki and B. V. Shanabrook, Semiconductors and Semimetals, Vol. 36, edited by D. G. Seiler and C. L. Littler (Academic, New York, 1992), p. 221.

[25] W. M. Theis, G. D. Sanders, C. E. Leak, K. K. Bajaj, and H. Morkoc, Phys. Rev. B 37, 3042 (1988).

[26] W. S. Yun, S. W. Han, S. C. Hong, I. G. Kim, and J. D. Lee, Phys. Rev. B 85, 033305 (2012).

[27] M. Rohlfing, Phys. Rev. B 82, 205127 (2010).

[28] M. Rohlfing and S. G. Louie, Phys. Rev. B 62, 4927 (2000).

[29] H. Jiang, J. Phys. Chem. C 116, 7664 (2012).

[30] X.-X. Zhang, Y. You, S. Y. F. Zhao, and T. F. Heinz, Phys. Rev. Lett. 115, 257403 (2015).

[31] J. P. Echeverry, B. Urbaszek, T. Amand, X. Marie, and I. C. Gerber, Phys. Rev. B 93, 121107(R) (2016).

[32] G. Wang, C. Robert, M. M. Glazov, F. Cadiz, E. Courtade, T. Amand, D. Lagarde, T. Taniguchi, K. Watanabe, B. Urbaszek, and X. Marie, Phys. Rev. Lett. 119, 047401 (2017).

[33] T. Deilmann and K. S. Thygesen, Phys. Rev. B 96, 201113 (2017).

[34] J. Horng, T. Stroucken, L. Zhang, E. Y. Paik, H. Deng, and S. W. Koch, arXiv:1712.04485.

[35] A. Arora, M. Koperski, K. Nogajewski, J. Marcus, C. Faugerasa, and M. Potemski, Nanoscale 7, 10421 (2015). 